\title{
Jubilación: Una transición ocupacional con consecuencias en la temporalidad, equilibrio y significado de la ocupación $\left({ }^{*}\right)$
}

Hans Jonsson (1), Lena Borell (2), Gaynor Sadlo (3)

\begin{abstract}
- Resumen
El propósito de este estudio era explorar la jubilación como una transición ocupacional. Veintinueve participantes de 66 años de edad fueron entrevistados y sus datos analizados utilizando un método comparativo cualitativo. El análisis mostró que se desarrolla una nueva estructura temporal en la que los participantes funcionan a ritmos más lentos. Algunas ocupaciones también cambiaron su significado cuando fueron realizadas en la nueva circunstancia de la jubilación. Un patrón común en la transición fue pasar de un desequilibrio, donde el trabajo ocupaba mucho tiempo de la vida, a otro tipo de desequilibrio donde algún tipo de compromiso regular post jubilación habría sido preferible. La discusión relata los hallazgos relativos al significado y ritmo de los conceptos en la dinámica de la teoría de sistemas. La importancia de los compromisos regulares en la vida para experimentar equilibrio ocupacional y la perspectiva cambiante de futuro también forman parte de la discusión.
\end{abstract}

Palabras clave.: Adaptación temporal, Ritmo ocupacional, Métodos cualitativos

\begin{abstract}
- Abstract
The aim of this study was to explore retirement as an occupational transition. Twenty-nine participants aged 66 years were interviewed and the data analysed using a comparative qualitative method. The analysis showed that a new temporal structure developed where the participants were gliding into a slower rhythm. Some occupations also changed meaning when they were performed in the new circumstances of retirement. A common pattern in the transition was to go from one imbalance, where work took too much time in life, to another type of imbalance where some kind of regular commitment within retirement would have been preferable. The discussion relates the findings concerning meaning and rhythm to concepts in dynamic systems theory. The importance of regular commitments in life for experience of occupational balance and the changing perspective of the future are also discussed.
\end{abstract}

Key words: Temporal adaptation, occupational rythm, qualitative methods

(1) Ph.D., OT(reg), Associated Professor, Director of Master Program in Occupational Therapy, Karolinska Institutet, Department of NEUROTEC Division of occupational therapy, (2) OT (reg), Dr Med Sc, Associated Professor, Board Chairman of the Center of Caring Sciences, (3) SROT, Ph.D., Head Division of Occupational Therapy

Contacto > > Alfred Nobels allé 23 S-141 83 Huddinge Sweden Phone: +46 852483876 Fax: +46 8345014 Internet address: http://www.ki.se/cnsf/occther/hansj.htm E-mail: Hans.Jonsson@neurotec.ki.se 


\section{- Introducción}

Este estudio explora las experiencias de las personas en la transición ocupacional del trabajo a la jubilación. El transitar desde un trabajo de tiempo completo a una vida cotidiana en que el trabajo ya no es lo dominante, es un cambio predecible para la mayoría de las personas en Occidente, y que tiene muchas implicancias.

Una revisión de la literatura reveló la existencia de investigación limitada que registrara la experiencia de las personas acerca de este cambio en su vida ocupacional diaria. El comprender estas experiencias puede sugerir caminos para apoyar a las personas que están próximas a jubilar. Este nuevo conocimiento también puede proveer una comprensión más profunda de cómo la forma, función y significado de la ocupación cambian en la medida que la persona realiza cambios de un patrón ocupacional a otro.

Como parte de un estudio longitudinal que utilizó métodos de investigación cualitativos, 29 participantes de 65-66 años de edad fueron entrevistados. Un método comparativo constante fue utilizado en el análisis. Cinco temas emergieron: nueva estructura temporal; entrando en un ritmo más lento; cambios en el significado; la paradoja de ser el "dueño" de tu propio tiempo; y la manutención de la estabilidad como el deseo principal para el futuro.

(*) Trabajo inicialmente publicado en: Journal of Occupational Science 2000; 7: 5-13. La presente publicación en castellano ha sido autorizada por el editor original.

Traducción técnica y revisión final: Ma Elena Riveros. Terapeuta Ocupacional. Escuela de Terapia Ocupacional, Universidad de Chile.

Primera traducción: Sr. Felipe Muñoz Cornejo

\section{- Revisión de literatura}

La ocupación es el foco central para la ciencia de la ocupación y ha sido propuesta como fundamento para la Terapia Ocupacional $(\underline{1}, \underline{2})$. Los presupuestos filosóficos acerca de las ocupaciones han sido formulados por diferentes estudiosos en el campo $(\underline{1}, \underline{3}-\underline{5})$, asumiendo la relevancia de la ocupación para la vida humana, el desarrollo y la salud. Basado en estos presupuestos, se han propuesto conceptos teóricos y modelos relacionados con la ocupación.

Uno de los conceptos clásicos de la Terapia Ocupacional es el equilibrio ocupacional, que ha sido definido tradicionalmente como el equilibrio de tiempo entre trabajo, juego, descanso y sueño en la vida humana. La literatura reciente revela otros aspectos del equilibrio ocupacional. Christiansen propuso el desarrollo de una visión del equilibrio focalizada en la relación entre tareas de la vida, mientras que Wilcock releva la naturaleza individual del equilibrio ocupacional. Se supone que el desequilibrio ocurre: "porque el involucramiento en ocupaciones falla en cubrir necesidades físicas, sociales, mentales o de descanso únicas.

El significado ocupacional es otro concepto central que ha sido discutido en la literatura ( $\underline{9}-\underline{12})$. Nelson definió el significado ocupacional como: el completo proceso interpretativo de una persona cuando enfrenta una forma ocupacional, incluyendo la experiencia perceptual, simbólica y afectiva $(\underline{13})$.

Espiritualmente ha sido vista como una expresión del significado de la existencia y del propósito de cada uno en la vida $(\underline{14}, \underline{15})$. Los individuos experimentan y expresan significado en sus vidas debido a sus ocupaciones. 
Como esta discusión lo demuestra, existe un emergente y creciente interés por el desarrollo de una comprensión conceptual de la ocupación humana. Sin embargo, el conocimiento generado por estudios empíricos es limitado y se ha focalizado en el significado experimentado en las diferentes ocupaciones (16). Muy pocos estudios empíricos han investigado cómo los humanos experimentan y orquestan su completa vida ocupacional (17-21). En un trabajo anterior, el primer autor propuso varias razones de por qué la jubilación provee de una valiosa oportunidad para estudiar la ocupación humana. En primer lugar, la jubilación representa un importante cambio en el ciclo vital. En segundo lugar, es un proceso que puede ser estudiado a lo largo del tiempo. En tercer lugar, durante el proceso de cambio las personas pueden tomar conciencia más fácilmente de los diferentes significados de la ocupación en sus vidas. Por ejemplo, dos estudios del primer autor conducidos en el periodo anterior a la jubilación, encontraron que era fructífero examinar los significados que las personas atribuían al trabajo cuando éste estaba por llegar a su fin $(\underline{23}, \underline{24})$. Los participantes, anticipándose a la jubilación, adoptaban diferentes actitudes hacía ésta dependiendo de los valores que atribuían al trabajo, y en la medida que percibían que otras ocupaciones con similar valor podrían reemplazar a éste. También ha habido pocos estudios relativos a la transición de trabajador a jubilado desde la perspectiva ocupacional real. Los estudios de la jubilación y ocupación se han focalizado principalmente, ya sea en la importancia de la preparación para la jubilación, o en la experiencia de satisfacción en una vida post jubilación.

En el campo de la gerontología social, la jubilación ha sido el foco de numerosos estudios cuantitativos, y un número menor de estudios cualitativos, aún así los resultados han sido inconsistentes $(\underline{22}, \underline{31})$. Varios estudios muestran una conexión general entre el número de actividades desarrolladas y la experiencia de satisfacción con la vida al momento de la jubilación. Sin embargo, la idea de que algunos tipos de actividad (ej. sociales) tiene una influencia mayor en la satisfacción vital que otras actividades (ej. no sociales) es apoyada por algunos estudios, pero no por otros (22). De manera similar, la existencia de una fase denominada de luna de miel justo después de la jubilación es apoyada por algunos estudios, mientras que otros señalan lo opuesto (31). Mein y colaboradores, compararon información cualitativa sobre jubilación con cuatro teorías sociológicas dominantes acerca de jubilación y adultos mayores (32): la teoría de la dependencia estructurada; la teoría del desenganche; la teoría de la tercera edad; y la teoría de roles. Encontraron apoyo mixto para cada una, pero concluyeron que todas eran demasiado reduccionistas para cubrir la naturaleza multifacética de la jubilación. Otros han argumentado que las teorías que lideran en sociogerontología a veces simplifican un proceso complejo cuando intentan establecer parámetros generales ( $\underline{3}-\underline{36})$.

Entonces, la investigación previa en jubilación nos muestra la necesidad de contar con nuevas perspectivas sobre esta fase de la vida humana, así como la necesidad de generar conocimiento empírico acerca de la jubilación desde una perspectiva ocupacional. Este estudio exploró cómo personas de 65-66 años que habían jubilado o se encontraban transitando hacia ésta, ocupaban su tiempo, y cómo experimentaban la jubilación como una transición ocupacional.

\section{- Método}

Para este estudio se escogió una metodología con base teórica (37- $\underline{39})$. Para explorar en profundidad un proceso en que los participantes estaban transitando a la jubilación se requería de entrevistas relativamente extensas. La estructura de las entrevistas se basaba en la noción de que el proceso de la jubilación podía ser investigado a través de la recolección de información de cómo los participantes ocupaban su tiempo y cómo se experimentaba ahora la vida diaria. El análisis de las entrevistas fue conducida también de una manera exploratoria, comparando datos del antes y el después en orden a cubrir los patrones de temas. 


\section{- Participantes}

Este estudio es parte de un estudio longitudinal sobre el proceso de jubilación experimentado por un grupo de personas que viven en una región socioeconómicamente diversa de Estocolmo, Suecia. Se escogieron de manera randomizada 32 personas de un registro público de la región de todas las personas de 63 años que se encontraban trabajando $50 \%$ o más. La participación fue voluntaria. El estudio siguió a los participantes por siete años, desde los 63 años, cuando se encontraban trabajando, a través de su jubilación o semi-jubilación. Este estudio en particular involucró 29 participantes cuando tenían 65-66 años, y la mayoría se había jubilado 6 a 12 meses antes.

Las condiciones básicas demográficas y de vida de los participantes se muestran en la tabla 1.

Tabla 1: Características demográficas de los participantes.

\begin{tabular}{|l|l|l|l|}
\hline & Total & Hombres & Mujeres \\
\hline Género & 29 & 15 & 14 \\
\hline & & & \\
\hline Condiciones Familiares & & & \\
\hline Vive con una pareja & 18 & 11 & 7 \\
\hline Vive solo & 11 & 4 & 7 \\
\hline Tiene hijos & 23 & 11 & 12 \\
\hline Tiene nietos & 18 & 8 & 10 \\
\hline & & & \\
\hline Condiciones de vida & & & \\
\hline Vive en departamento & 26 & 14 & 12 \\
\hline Vive en casa propia & 3 & 2 & 1 \\
\hline Tiene casa de veraneo & 18 & 11 & 7 \\
\hline \hline & & & \\
\hline Tipo de trabajo cuando trabajaban & & & \\
\hline Mando medio o inferior & 20 & 8 & 12 \\
\hline Mando medio o superior & 9 & 7 & 2 \\
\hline & & & \\
\hline Estatus al jubilar & & & \\
\hline Jubilación de tiempo completo & 19 & 9 & 10 \\
\hline Trabajo de tiempo parcial & 8 & 4 & 4 \\
\hline Trabajo de tiempo completo & 2 & 2 & 0 \\
\hline
\end{tabular}

\section{- Recogida de datos}

El autor principal condujo las entrevistas de carácter informal y semiestructurado. El tema completo era descubrir cómo las cosas habían cambiado para los participantes en la medida que redujeron horas de trabajo o se jubilaron. Se desarrolló una guía para las entrevistas investigando los 
siguientes temas: a) cómo se experimentó el primer día de la jubilación, b) pensamientos personales acerca de ser jubilado y proceso de adaptación, c) descripción de una semana ordinaria como jubilado y similitudes y diferencias comparado con una semana cuando se encontraba trabajando, d) pensamientos acerca de su futuro como jubilado, e) una opinión completa acerca de la transición de trabajador a jubilado. Las entrevistas duraron entre 40 y 90 minutos. Las entrevistas fueron grabadas y luego transcritas para su análisis.

\section{- Análisis de datos}

Se usó una aproximación inductivo-cualitativa siguiendo los pasos del método comparativo ( $\underline{37}-\underline{39})$. En un proceso de codificación abierta al comienzo del análisis, los datos fueron categorizados en códigos sustantivos de acuerdo a los temas de la entrevista. En el siguiente paso se desarrollaron códigos teóricos basados en los códigos sustantivos. Un propósito en el análisis era descubrir patrones generales en los datos. En este paso el análisis fue hacia adelante y hacia atrás comparando los códigos emergentes, unos con otros. Cinco temas emergieron de esta comparación: nueva estructura temporal; entrando en un ritmo más lento; cambios en el significado; la paradoja de ser el "dueño" de tu propio tiempo; y la manutención de la estabilidad como el deseo principal para el futuro.

El tema de la credibilidad fue abordado en este trabajo basándose en los trabajos de Lincoln y Guba (40). Todos los temas que emergieron desde los datos fueron revisados en conjunto con pares investigadores experimentados, familiarizados con el análisis comparativo. Esto aseguró que todos los temas se basaran en datos obtenidos en la entrevista. Los temas se presentan en este trabajo con citas de las entrevistas para demostrar y confirmar la exactitud de cada uno de ellos. Las entrevistas fueron realizadas en sueco y las traducciones al inglés fueron asumidas por el investigador principal y luego discutidas con el grupo de investigadores.

\section{- Hallazgos}

Los hallazgos del estudio se relacionan con la experiencia de tiempo, significado y equilibrio de las ocupaciones en las cuales los jubilados se involucran. El aspecto temporal incluía nuevas estructuras temporales: un ritmo de vida más lento y ser el dueño de su propio tiempo. Las expectativas acerca del futuro, el significado de las ocupaciones y un nuevo equilibrio en la ocupación también aparecen como hallazgos claves. Estos son discutidos en detalle a continuación:

\section{- Nueva estructura temporal}

Cuando la persona ya no trabaja o cuando el trabajo ya no ocupa la mayor parte de su vida, como en el caso de la jubilación parcial, la completa estructura de la vida cotidiana obviamente cambia. Los participantes se declaran liberados de las demandas del trabajo y cómo ahora pueden planificar y agendar el día y la semana ellos mismos. Las ocupaciones que antes se realizaban por las tardes y los fines de semana, por ejemplo limpiar la casa y la lavandería, ahora se realizaban durante el día y en la semana. Hacer compras de día, hacer ejercicio, participar en cursos y juntarse con amigos era posible, lo que contrastaba fuertemente con las actividades realizadas durante el día cuando trabajaban. Las tardes se ocupaban para el "descanso", descrito como leer y ver televisión. En la nueva estructura semanal, el fin de semana era utilizado para relajarse, 
mientras que los días de la semana se encontraban más ocupados. Sin embrago, las ocupaciones que antes se restringían a los fines de semana, tales como ir a la casa de veraneo, ahora se realizaban durante toda la semana. Los participantes declararon que su estructura del tiempo ya no tenía actividades distintivas dentro de un ritmo semanal. Las diferencias entre días de semana y fines de semana eran ahora descritos como relacionados con (por ejemplo), encontrar menos tiendas abiertas en los fines de semana, y amigos del trabajo aún más disponibles los fines de semana. Algunos participantes refirieron dificultades para mantener contacto social con amigos que aún se encontraban trabajando.

Los participantes también encontraban difícil reponer las rutinas creadas externamente por el trabajo, cuando tenían que manejar las rutinas ellos mismos. Esto puede ser ejemplificado por un hombre que comenzó un negocio de consultora después de la jubilación; encontró dificultades en ser el quién regía su propio tiempo.

Ha sido difícil crear nuevas rutinas de trabajo... En 25 años yo sabía qué me regía y qué regía yo. Y me encontraba muy satisfecho y la vida transcurría. Pero ahora - para encontrar una nueva vida - una disciplina para hacer las cosas que quiero e imponérmelo a mi mismo toma tiempo y no estoy seguro si estoy listo para ello aún.

\section{- Transitando hacia un ritmo de vida más lento}

Los participantes describieron cómo el primer año como jubilados adoptaron un ritmo de vida diario más lento. La mañana era descrita como el periodo del día en que esto se hacía más evidente. Tomar desayuno y leer el periódico página por página tomaba casi toda la mañana. La transformación en un ritmo era descrita como un proceso de tránsito, un ajuste en el que entraron casi sin darse cuenta de ello, antes o durante este proceso. Algunos participantes se encontraban sorprendidos, cuando se reflejaban en la actual situación en relación a la situación que se encontraban antes de jubilar. Estos participantes se preguntaban a sí mismos cómo alguna vez tuvieron tiempo para hacer estas cosas que querían y necesitaban hacer, mientras se encontraban trabajando. Una participante nos ofrece su reflexión acerca del cambio de ritmo y revela una experiencia de perplejidad frente al tiempo disponible.

Cuando voy a hacer algo hoy me toma todo el día. Antes tenía tiempo para hacer varias cosas.

Pareciera ser que una nueva estructura temporal es creada después de la jubilación. La transición fue descrita como un proceso gradual que conduce a un ritmo de vida más lento. La mayoría de los participantes describe este proceso en términos positivos, con un sentimiento de ser dueño de su día. Expresiones tales como; "es más calmado ahora" y "es menos estresante" fueron utilizadas para describir el nuevo ritmo. Sin embargo, algunos participantes experimentaban su nuevo paso hacia un ritmo más lento como un vacío que no saben cómo llenar. Unos pocos habían tenido la esperanza de llenar el tiempo con ocupaciones significativas, pero habían encontrado dificultad para lograrlo.

Esto arroja luz sobre otro hallazgo relacionado con la adaptación temporal, que es que los participantes tienen planes de nuevas ocupaciones después de su jubilación. La mayoría de los participantes habían anticipado que comenzarían a realizar nuevas actividades, o retomarían actividades de su juventud, pero éstos eran aún más bien planes que una realidad. Y no había problemas de salud o económicos evidentes para no realizar estos posibles planes. Un participante sentenció: Sí, he estado pensando sobre ello, pero no he hecho nada aún. Cuando los participantes reflexionaron sobre ello, se sorprendieron de no tener tiempo para realizar sus planes. El hallazgo de que sólo unos pocos participantes empezaron a realizar nuevas actividades puede estar relacionado con el enlentecimiento del ritmo de vida cotidiano. Cuando los 
participantes trabajaban, esperaban tener un montón de tiempo disponible cuando jubilaran para desarrollar nuevas ocupaciones. Su ritmo enlentecido de vida, sin embargo, significó que el tiempo disponible para hacer nuevas actividades también disminuyó. Un ritmo más lento significa que se requiere mayor tiempo para desarrollar cada ocupación y los proyectos de realizar nuevas ocupaciones permanecían más bien como ideas e intenciones.

\section{Cambio en el significado}

Dentro de la nueva estructura temporal, algunos participantes describieron un cambio en el significado de las ocupaciones., o dificultad para experimentar el mismo significado que tenían antes de la jubilación. Un hombre que trabajaba tiempo completo cuatro días a la semana antes de jubilar declaró un cambio de significado en las idas a su casa de veraneo:

Era un verdadero prioridad ir a la casa de verano el jueves por la tarde después de haber trabajado cuatro días. Tenía el fin de semana completo para relajarme. Ahora no es lo mismo nunca más, no tengo el mismo sentimiento frente a ello... Da lo mismo si es domingo pues ahora no tenemos que volver a la ciudad. Y las diferencias entre fines de semana y días de la semana han desaparecido.

Esto describe un cambio en el significado para una vieja y bien conocida ocupación cuando era desarrollada dentro de otra estructura ocupacional. La estructura que el trabajo había creado antes de la jubilación influía en el significado experimentado en otras ocupaciones, una ocupación creaba condiciones importantes para otras. Esto se volvió obvio para los individuos cuando la antigua ocupación fue desarrollada en una nueva estructura diaria.

Los participantes también describieron un mecanismo de ayuda preexistente útil, puesto que las demandas del trabajo les ofrecian la posibilidad de escapar de otras demandas del medio social (por ejemplo, los hijos y la pareja) y de las obligaciones auto impuestas. En la jubilación, las obligaciones del trabajo ya no podían ser usadas como excusa. Por ejemplo, una mujer nos dijo:

Pienso que era más calmado cuando trabajaba. Porque entonces simplemente debía dejar algunas cosas de lado.

Los significados significativos dados a los tiempos y a los días de la semana, en una vida de trabajo estructurada, fueron descubiertas sólo cuando estas rutinas de trabajo se perdieron. Un hombre, quien antes de la jubilación, había estado añorando el día en que dejaría de trabajar, dijo:

Es una vida especial la que uno vive cuando tienes esto, trabajo por la mañana y los fines de semana libres. $Y$ existen los lunes por la mañana y todo eso. $Y$ como todo eso se ha ido de verdad lo echo de menos. Ahora los días no importan más, si es lunes por la mañana o viernes por la tarde.

Se encontró a sí mismo (para su sorpresa) echando de menos las rutinas del trabajo durante su jubilación. 


\section{La paradoja de ser el dueño de su propio tiempo}

De los participantes que se encontraban jubilados tiempo completo, algunos aún miraban su vida ocupacional como si se encontraran trabajando; cuidando nietos, haciendo las tareas diarias de la casa o cuidando la casa de veraneo. Sin embargo, esto no fue un patrón general en los datos, una misma tarea podía ser vista como trabajo o descanso. Un participante que tenía una casa de veraneo dijo:

Siento que ahora tengo descanso todo el tiempo. Supongo que cuando uno es un jubilado es el único tiempo de la vida que a uno le pertenece de verdad.

Ser dueño de su propio tiempo, significa que uno no está obligado a hacer ninguna cosa y puede decidir por uno mismo. Cuando los participantes se encontraban trabajando, el trabajo llenaba la mayor parte de su agenda diaria y otro tipo de actividades debía ser presionada dentro del tiempo que quedaba libre. Cuando el trabajo ya no estuvo presente en la vida diaria esto fue descrito como "ser el amo de su propio tiempo", un cambio preferible para la mayor parte de los participantes.

Sin embargo, la libertad de ser enteramente el dueño de su propio tiempo era experimentado de un modo paradojal. El análisis demostró un patrón en el que algunos jubilados deseaban tener más demandas y ocupaciones productivas que regularan y estructuraran su vida cotidiana. Los participantes experimentaban una pérdida de una parte preferida de la vida, definida como trabajo remunerado o como trabajo voluntario, a pesar de que no deseaban volver a la vida como era antes de jubilar. Lo que la mayoría de los participantes deseaban eran obligaciones regulares, descrito como la posibilidad de trabajar de uno a dos días y medio por semana, o unas pocas horas al día. Este deseo fue descrito así:

A mí me gustaría mucho, mucho, mucho tener un trabajo pequeño. No como en la vieja Compañía sino un trabajo pequeño que pudiera manejar. Así como cortar el pasto o los cercos. O salir a dar una vuelta o de compras con gente mayor.

Los participantes que no describieron este tipo de necesidad, o portaban condiciones de salud que los hacían pensar que una parte de vida productiva no era una posibilidad realista para ellos, o ya se encontraban altamente involucrados en actividades de tiempo libre/obligaciones familiares. Estos enganches eran en su mayoría obligaciones regulares, tales como estar en la Dirección de una organización de tiempo libre, los que son referidos en términos similares al trabajo y presentan condiciones como las de un trabajo.

Los participantes que describieron una falta de trabajo en su jubilación tenían pensamientos acerca de posibles modos de llenar esta carencia, tales como trabajo voluntario, o trabajar cuando fuera necesario en su antiguo trabajo. Uno de los argumentos más sólidos para no dar crédito a planes de trabajo remunerado era que una persona mayor no debiera ocupar el trabajo que una persona joven requiere, especialmente cuando el desempleo era alto (estaba en aumento durante la época de las entrevistas, y era una situación mencionada comúnmente por los participantes). Una mujer expresó su visión de la siguiente manera:

Por qué sólo debo andar por ahí sin hacer nada, pienso. Merezco tener un verdadero trabajo. Pero, por otro lado pienso por qué debiera trabajar yo, cuando hay tanta gente sin trabajo.

Sentimientos de estar "amarrados" influyeron en la decisión de si buscar o no algún tipo de trabajo. Una mujer dijo que tenía planes de hacer algún tipo de trabajo voluntario, pero ella también quería libertad para tener la posibilidad de viajar sin previo aviso para ver a su hijo y nietos. 
El grupo de participantes que aún mantenía algún tipo de trabajo valoraba esto muy positivamente. Un hombre que trabajaba $30 \%$ de su tiempo explicó su visión de ser el dueño de su propio tiempo:

Tengo mi tiempo, es mío. Puedo usarlo - y no quiero usarlo sólo como tiempo libre - quiero usarlo activamente- y es muy grato sentir esto. Sí, eso es libertad.

Otro ejemplo es una mujer que trabajaba cerca de medio tiempo:

Realmente tuve una situación ideal durante este tiempo. No ser cortada de inmediato, sino que ir dejando de a poco es una ventaja. Por otra parte, trabajo tiempo completo un día y el día siguiente no trabajo nada.

Al preguntar por las razones para trabajar, los participantes expresaron una necesidad de tener más demandas de la sociedad o desde fuera de sí mismos, a menudo expresado en frases como: "teniendo algo organizado para hacer" o "uno se siente más o menos feliz de sentirse necesitado, eso es verdad". La oportunidad de usar el conocimiento, las destrezas y las experiencias que las personas han adquirido durante su vida laboral eran también valorados. Además, los contactos sociales en general y conectados con resolución de problemas en el trabajo, fueron descritos.

\section{- Mantener la estabilidad como el deseo principal para el futuro}

En las entrevistas los participantes fueron interrogados acerca de sus planes para el futuro. La ausencia de proyectos a largo plazo fue un patrón general en los datos; los proyectos para el futuro se limitaban a la próxima estación o hasta el próximo año solamente. Otro hallazgo fue que los participantes enfatizaron que realmente deseaban "que las cosas se mantuvieran igual". El deseo parecía estar directamente relacionado con la salud, tal como: "en la medida en que pueda mantener mi salud todo lo demás está bien".

Mantener la salud no era sólo un deseo sino que un propósito puesto en acciones concretas. Muchos de los participantes hacían algún tipo de actividad física regular con el objetivo de mantenerse en forma. Un participante explicó sus motivaciones. "Todos los jubilados soñamos con no enfermarnos. Por lo menos trato de mantener mi físico y hago lo que puedo para no dañarlo". Lo más común para mantenerse en forma era la caminata diaria, solitaria, con una pareja, un amigo o juntos en un grupo de tercera edad. Los participantes también desarrollaban distintos tipos de deportes tales como aeróbicos y correr.

En general, los participantes parecían haber adoptado una perspectiva de que habían entrado o estaban por entrar en un periodo de sus vidas en que se va lentamente hacia abajo. Desde esta perspectiva, la salud, incluyendo tanto lo físico como las capacidades generales, era esperable que fueran disminuyendo poco a poco. Los jubilados temían perder la capacidad para moverse libremente, o que llegarían a tener serios problemas intelectuales. Las personas se encontraban ansiosas no sólo por ellas mismas, sino por sus parejas y amistades. Desde la perspectiva de "cuesta abajo", su deseo era que nada cambiara, y que su salud permaneciera igual. También parecía lógico no hacer planes a largo plazo. Los participantes que vivían con una pareja conceptualizaban el mantener la salud a futuro como un objetivo compartido, y aquellos que tenían problemas de salud tenían la misma perspectiva. Una mujer con problemas de salud declaró:

Había decidido, esto es como las cosas están y estarán a futuro, y estaremos agradecidos si las cosas no se vuelven peor de lo que están hoy. 


\section{- Resumen de los hallazgos}

Veintinueve participantes fueron entrevistados cuando tenían alrededor de 66 años de edad la mayoría jubilados o semi-jubilados. El proceso de jubilación fue diverso - pasaron a la jubilación y describieron las experiencias acerca de ésta de manera distinta. A pesar de que la diversidad fue un aspecto importante en este estudio, temas generales de importancia y patrones comunes pudieron ser descubiertos en los datos obtenidos.

Se desarrolló una nueva estructura ocupacional donde encontrarse con amigos, hacer ejercicio, participar en intereses, y las actividades domésticas tuvieron cabida durante el día y durante la semana, mientras que las tardes y los fines de semana se focalizaban más en actividades de relajo. Algunas ocupaciones también cambiaron su significado, y sus significados previos sólo fueron descubiertos cuando las viejas estructuras de vida se perdieron. Es aparente que las ocupaciones no sólo tienen significado por sí mismas, sino que en relación a otras ocupaciones, y en la completa organización de la vida.

Junto con la nueva estructura ocupacional, se desarrolla un ritmo más lento de vida con la jubilación. La mayoría de los participantes describieron estos cambios en términos positivos, como estando en un ritmo menos estresante y teniendo un sentimiento de ser el dueño de su propio tiempo. El transitar hacia un ritmo más lento también significaba que el tiempo disponible para nuevas ocupaciones fue inesperadamente experimentado como disminuido.

La situación de jubilación fue experimentada como positiva, pero al mismo tiempo como si faltara una parte de la vida. La transición puede ser vista como transitando desde un desequilibrio, donde el trabajo ocupaba demasiado tiempo, a otro tipo de desequilibrio donde tener algún tipo de obligaciones regulares habría sido deseable durante la jubilación. Un número de factores individuales y ambientales influyeron en el descubrimiento de que el querer hacer más a menudo fracasa en llegar a ser realidad. Generalmente, se ocupaba más tiempo en realizar las tareas diarias ordinarias, desplazando el tiempo para emprender nuevas ocupaciones. Otro factor fue la perspectiva de los participantes respecto a su futuro. Esta era una perspectiva de "cuesta abajo" donde la mayor esperanza para el futuro es que las cosas permanecieran igual. Los planes a futuro no se extendían más allá del año. El mantener la salud era lo más importante, y llevado a cabo en acciones concretas.

\section{- Discusión}

Esta estudio demuestra que una transición ocupacional tal como la jubilación es un cambio que tiene un amplio impacto en varios aspectos de la vida. La jubilación puede ser vista como un ejemplo de una transición ocupacional mayor y usualmente abrupta.

La nueva estructura temporal de la vida diaria demostrada en este estudio no es un cambio sorprendente en la jubilación, dado que una ocupación de larga data ha llegado a su fin (a pesar de que a veces no sea escogido). Encontrar lo contrario habría sido inesperado. A pesar de esto, el hallazgo de que las nuevas ocupaciones no siempre llenaban el vacío dejado por el trabajo es notable, y es relevante para la preparación para la jubilación. Esto debiera ser explorado con mayor profundidad en otras investigaciones. Un hallazgo importante en este estudio es que los significados de las ocupaciones pueden cambiar en esta nueva estructura. El significado de la ocupación ha sido planteado como algo muy relevante para comprender la ocupación humana ( $\underline{9}-$ 12). Previamente, los significados han sido vistos como provenientes de la experiencia interna del sujeto, lo que ha sido confirmado en parte en este estudio. Sin embargo, los resultados aquí también demuestran que el significado de una ocupación está determinado en parte por su relación con otras ocupaciones - tales como el cambio en el significado de ir a la casa de veraneo después 
de la jubilación. Más que ser sólo intrínseca de la persona (o la ocupación) estos significados ocurren en la relación que constituyen las circunstancias para cada ocupación.

Estos hallazgos coinciden con aspectos de la teoría general de sistemas, que ha sido identificada como un marco teórico útil para conceptualizar la Ocupación Humana (41-43). La dinámica de la teoría de sistemas asume que la ocupación se caracteriza por una interacción continua en una compleja red (42). El desarrollo de un sistema altamente complicado es impredecible y no lineal. El significado en la ocupación humana puede, desde una perspectiva dinámica sistémica, ser visto como el resultado de una interacción no lineal entre muchos factores en la red de un sistema complejo. Cuando un individuo se plantea frente a un cambio tan importante como es la jubilación, es difícil pensar cómo el trabajo afecta el significado de otras ocupaciones, y esto se vuelve obvio sólo cuando se experimenta un cambio en las circunstancias.

Otro hallazgo relacionado con el concepto de la dinámica de sistemas es el enlentecimiento del ritmo de vida con la jubilación. Este hallazgo podría ser visto en el contexto de un sistema complejo que subyace a los cambios y a la auto-organización espontánea en un nuevo patrón ocupacional con un nuevo ritmo (42). Cuando el trabajo era parte del sistema mantenía el ritmo (demasiado alto a veces). Cuando el trabajo desaparece, el ritmo se hace más lento, un resultado que sorprende a los individuos, puesto que no se trataba de una decisión consciente. Sin las demandas de tiempo que comprimen las tareas diarias, tales como comer o ponerse al día con las noticias, éstas tomaban mucho más tiempo, llenando la mayor parte del día y dejando menos tiempo para las ocupaciones que los jubilados pensaban que harían. Tal vez este es un factor de la jubilación reciente, del ajuste para llegar a tener más tiempo libre. Las ocupaciones de supervivencia y ocio de cada día parecían ser disfrutables por sí mismas, cuando antes debían ser realizadas muy a la rápida. La forma ocupacional de algunas actividades rutinarias, tales como preparar desayuno o leer el periódico de la mañana, obviamente cambian durante la jubilación, un hallazgo que podría ser explorado más allá.

Los participantes describían el cambio de ritmo como un proceso de transición que los sorprendía. Ellos miraban hacia atrás desde este nuevo horizonte con un ritmo más lento y se preguntaban cómo habían tenido tiempo para trabajar tiempo completo. Se encontraban en un nuevo patrón sin tener clara conciencia de ello. El concepto de auto-organización en sistemas dinámicos sugiere que cuando los sistemas se desestabilizan, ellos tienden a sobrellevar cambios espontáneos para organizarse a sí mismos a menudo de formas inesperadas (42). Este concepto transforma la sorpresa acerca del cambio del ritmo en la jubilación en algo más comprensible y sugiere también que éste podría ser de la adaptación ocupacional al cambio.

En general, para la mayoría de los participantes, la jubilación significaba libertad y ser el dueño de su propio tiempo. Varios estudios han encontrado que algunas pocas personas durante su jubilación desean volver al trabajo que tenían antes $(22,24,31)$ pero un hallazgo importante en este estudio es que la libertad era experimentada de una manera paradojal. Los individuos en este estudio añoraban la libertad de dejar de trabajar, y cuando se jubilaron la disfrutaban, pero al mismo tiempo tenían el deseo de tener una parte productiva en sus vidas, algún tipo de responsabilidad de carácter regular. Sin esto se encontraban transitando desde un desequilibrio en sus vidas hacia otro. Un ejemplo de lo opuesto eran los participantes que ahora trabajaban un $30 \%$ y valoraban esto como la libertad última que les permitía un buen equilibrio de sus ocupaciones durante la jubilación.

La mayoría de los participantes en este estudio que tenían en mente hacer algún tipo de trabajo no lo habían concretado debido a diferentes circunstancias individuales y contextuales. Esta intención concuerda con un estudio mayor realizado en Suecia por el primer autor donde $73 \%$ del grupo de personas de 63 años de edad tenían actitudes positivas hacia el trabajo ya fuera voluntario o remunerado durante la "jubilación" (44). El que no tuvieran aún implementados su planes concuerda con las estadísticas nacionales suecas (44), lo que demuestra que pocos jubilados llevan a cabo sus planes. Esto tiene implicancias en las decisiones respecto a políticas, por el bien 
tanto de la sociedad como del individuo. La importancia de tener responsabilidades regulares durante la jubilación es clave en relación con el equilibrio y desequilibrio ocupacional y se conecta cercanamente con la experiencia de ocupación saludable. El concepto de flujo, donde las experiencias óptimas se caracterizan por altos desafíos que coinciden con el uso máximo de habilidades personales puede también iluminar los hallazgos del estudio. La teoría de flujo sugiere que cuando las ocupaciones desafiantes, que coinciden con las habilidades del individuo, no están presentes, el resultado será una atrofia emocional. El deseo de tener responsabilidades regulares durante la jubilación puede ser visto como un desequilibrio ocupacional, donde los desafíos no coinciden con las habilidades del individuo.

El deseo de tener obligaciones regulares también tiene implicancias para las políticas de jubilación de una sociedad. Todas las sociedades occidentales enfrentan un gran aumento de población adulta que inicia su jubilación. Una implicancia de esto es que se requiere un abordaje más flexible acerca del tema. Otra es que en función de proveer salud, la política de la sociedad debiera ser fomentar algún tipo de responsabilidad como parte de ser un jubilado. Tal política podría combinar la satisfacción individual con la utilidad social.

Los hallazgos de que los jubilados perciben la jubilación como un periodo de lenta declinación demuestra la importancia de comprender la manera en que la gente mayor anticipa el futuro. Desde la perspectiva de la declinación inevitable, parece comprensible no tener objetivos ocupacionales más extensivos. Mantener las cosas más o menos de la misma manera parece convertirse en una visión positiva para el futuro. Desde la perspectiva de una persona joven esto puede aparecer como un objetivo negativo, sin embargo es importante reconocer que esto ocurre para así entender la perspectiva de las personas mayores.

Este estudio no reveló ninguna relación entre características sociodemográficas y los hallazgos. Por ejemplo, el género, tipo de trabajo, y estatus marital no pudieron ser relacionados con los patrones descubiertos en los datos. En una muestra mayor podría ser posible estudiar esa relación, pero es importante reconocer que los cuadros estereotipados de las personas, tomados de ejemplos mayores, pueden no ser aplicables a individuos.

\section{- Limitaciones del estudio}

En general, este estudio identificó que para este grupo de jubilados suecos, la experiencia de la jubilación estaba limitada a un contexto. Los estudios sobre jubilación en otras culturas ayudarían a comprender cuáles son los patrones generales en la transición a la jubilación y qué aspectos se encuentran limitados al contexto cultural. Los estudios de otras transiciones ocupacionales, tal como la transición del rol de estudiante al trabajo, desempleo para trabajar, o regreso al trabajo después de la enfermedad también contribuiría a la comprensión de la experiencia de las transiciones ocupacionales en general.

El tamaño de este estudio hizo posible analizar los datos con un método cualitativo comparativo y descubrir algunos patrones generales importantes así como aspectos particulares de estos patrones. Así como la heterogeneidad es un aspecto importante de los estudios sobre jubilación, estudios en profundidad que profundicen los conceptos del proceso de jubilación también se necesitan. Tal vez la limitación más importante de este estudio es que se examina una parte de un proceso que es un continuo. Por ejemplo, no se sabe si la experiencia de la transición ocupacional de la jubilación se mantendrá estable después de 5 o 10 años. Además alrededor de un tercio de los participantes tenían algún tipo de trabajo al momento de realizarse el estudio. Muchos de estos participantes se encontraban en el proceso de una transición gradual o postpuesta. ¿Experimentarán ellos los resultados de la transición de una manera diferente? Comprender la jubilación como un proceso en desarrollo es importante, pero se encontraba fuera de los objetivos 
de este estudio. Como este estudio forma parte de un proyecto de investigación longitudinal acerca de la jubilación, es probable que otros aspectos de la jubilación, como proceso de desarrollo ocupacional, se hagan evidentes en estudios futuros.

\section{Referencias}

1 Wilcock A A. An occupational perspective on health. Thorofare, NJ: Slack Incorporated, 1998.

2 Christiansen C, Clark F, Kielhofner G, Rogers J, Nelson D. American Occupational Therapy Association. Position paper: Occupation. American Journal of Occupational Therapy 1995: 49(10): 1015-8.

3 Reilly M. Occupational Therapy can be one of the great ideas of $20^{\text {th }}$ century medicine. Eleanor Clarke Slagle Lecture. American Journal of Occupational Therapy 1962: 16: 1-9.

4 Yerxa EJ. Health and the human spirit for occupation. American Journal of Occupational Therapy 1998: 52(6): 412-22.

5 Kielhofner G. Conceptual foundations of Occupational Therapy (2 $2^{\text {nd }}$ ed.). Philadelphia: F.A. Davis Company, 1997.

6 Meyer A. The philosophy of Occupational Therapy. American Journal of Occupational Therapy 1922. Reprinted 1977:31(10): 639-642.

7 Christiansen $\mathrm{CH}$. Three perspectives on balance in occupation. In: Zemke R, Clark F (editors). Occupational science. The evolving discipline. Philadelphia: F.A. Davis Company, 1996:431-451.

8 Wilcock, 1998, p.138.

9 Kielhofner G. Borell L, Burke J, Helfrich C, Nygard L. Volition subsystem. In: Kielhofner G. (editor). A model of human occupation: Theory and application $\left(2^{\text {nd }}\right.$ edition). Baltimore: William 6 Wilkins, 1995: 39-62.

10 Nelson DL. Therapeutic occupation: a definition. American Journal of Occupational Therapy 1996: 50(10): 775-82.

11 Jackson J. Is there a place for role theory in occupational science?... part 2. Journal of Occupational Science 1998: 5(2): 56-65.

12 Crabtree JL. The end of Occupational Therapy. American Journal of Occupational Therapy 1998: 52(3): 205-14.

13 Nelson, 1996, p.776.

14 Towsend E (editor). Enabling occupation. An Occupational Therapy perspective. Ontario. Canadian Association of Occupational Therapists, 1997.

15 Kirsh B. A narrative approach to addressing spirituality in Occupational Therapy. Canadian Journal of Occupational Therapy 1996: 63(1): 55-61.

16 Rudman DL, Cook JV, Polatajko H. Understanding the potential of occupation: A qualitative exploration of senior's perspectives on activity. American Journal of Occupational Therapy 1997: 51(8): 640-50.

17 Hannam D. More than a cup of tea: Meaning construction in an everyday occupation. Journal of Occupational Science Australia 1997: 4(2): 69-74.

18 Ludwig FM. How routine facilitates well-being in older women. Occupational Therapy International 1997: 4(3): 213-28. 
19 Nygard L, Borell L. A life-world of altering meaning: Expressions of the illness experience of dementia in everyday life over three years. Occupational Therapy Journal of Research 1998: 18(2): 109-36.

20 Clark F. 1993 Eleanor Clarke Slagle Lecture. Occupational embedded in a real life: Interweaving occupational science and Occupational Therapy. American Journal of Occupational Therapy 1993: 47(12): 1067-78.

21 Helfrich C, Kielhofner G. Volitional narratives and the meaning of therapy. American Journal of Occupational Therapy 1994: 48(4): 319-26.

22 Jonsson $\mathrm{H}$. The retirement process in an occupational perspective: A review of literature and theories. Physical \& Occupational Therapy in Geriatrics 1993: 11(4): 1534.

23 Jonsson $\mathrm{H}$, Kielhofner G, Borell L. Anticipating retirement: the formation of attitudes and expectations concerning an occupational transition. American Journal of Occupational Therapy 1997: 51(1): 49-56.

24 Jonsson H, Andersson L. Attitudes to work and retirement. Generalization or diversity? Scandinavian Journal of Occupational Therapy 1999: 61(1): 29-35.

25 Kendall A. Comment. Preparation for retirement: The occupational perspective. Journal of Occupational Science Australia 1996: 3(1): 35-8.

26 Cantor S. Occupational therapists as members of preretirement resource teams. American Journal of Occupational Therapy 1981: 35: 638-643.

27 Boderrick T, Glazer B. Leisure participation and the retirement process. American Journal of Occupational Therapy 1983: 37: 15-22.

28 Tincher BJV. Retirement: perspectives and theory. Physical \& Occupational Therapy in Geriatrics 1992: 11(1): 55-62.

29 Oakley C, Pratt J. Voluntary work in the lives of post-retirement adults. British Journal of Occupational Therapy 1997: 60(6): 273-6.

30 Gregory MD. Occupational behaviour and life satisfactions among retirees. American Journal of Occupational Therapy 1983: 37: 548-553.

31 Gall TL, Evans DR, Howard J. The retirement adjustment process: Changes in the well-being of male retirees across time. Journal of Gerontology 1997: 52 B(3): 110 117.

32 Mein G, Higgs P, Ferrie J, Stansfeld SA. Paradigms of retirement: The importance of health and ageing in the Whitehall II study. Social Science \& Medicine 1998: 47(4): 535-545.

33 Hendricks J. The search for new solutions. The Gerontologist 1996: 36(2): 141-144.

34 Grigsby JS. The meaning of heterogeneity: An introduction. The Gerontologist 1996: 36(2): 145-146.

35 Calasanti TM. Incorporatin diversity: Meaning levels of research, and implications for theory. The Gerontologist 1996: 36(2): 147-156.

36 Light JM, Grigsby JS., Blight MC Aging and heterogeneity: Genetics, social structure and personality. The Gerontologist 1996: 36(2): 165-173.

37 Bodgan RC, Bilken SK. Qualitative research for education - An introduction to theory and methods ( $2^{\text {nd }}$ edition). Needham heights, MA: Allyn and Bacon, 1992.

38 Glaser B, Strauss AL. The discovery of grounded theory: Strategies for qualitative research. Chicago: Aldine, 1967. 
39 Glaser B. Theoretical sensitivity: Advances in the methodology of grounded theory. Mill Valley, CA: Sociology Press, 1978.

40 Lincoln L, Guba EA. Naturalistic inquiry. Beverly Hills, CA: Sage, 1985.

41 Kielhofner G. Human systems. In: Kielhofner G. (editor). A model of human occupation: Theory and application ( $2^{\text {nd }}$ edition). Baltimore: Williams \& Wilkins, 1995: 9-25.

42 McLoughlin Gray J, Kennedy BL, Zemke R. Application of dynamic systems theory to occupation. In: Zemke R, Clark F (editors). Occupational science: The evolving discipline. Philadelphia. F.A. Davis Company, 1996: 309-324.

43 Baum C, Christiansen C. The Occupational Therapy context - philosophy - practice. In: Christiansen C, (editor) Occupational Therapy: Enabling function and well-being $\left(2^{\text {nd }}\right.$ edition) Thorofare, NJ: Slack Incorporated, 1997: 27-45.

44 Statistics Sweden. Arbetskraftssundersökningen, ärsmedeltal 1992. Labor Force Investigation, Yearly Average 1992). Stockholm: Statistiska Centralbyrän, 1993.

45 Csikszentmihalyi M. Play and intrinsic rewards. Journal o Humanistic Psychology 1975:15: 41-63.

46 Csikszentmihalyi M. Finding flow: The psychology of engagement with everyday life. New York: Basic Books, 1998. 